\title{
Tradução, Adaptação e Evidências de Validade do Thinking Styles Inventory - Revised II (Tsi-R2) no Brasil
}

\author{
Katya Luciane de Oliveira ${ }^{1}$ \\ Universidade Estadual de Londrina, Londrina-PR, Brasil \\ Acácia Aparecida Angeli dos Santos, Amanda Lays Monteiro Inácio \\ Universidade São Francisco, Campinas-SP, Brasil
}

\section{RESUMO}

O objetivo deste estudo foi traduzir, adaptar e analisar evidências de validade de conteúdo para o Thinking Styles Inventory - Revised II (TSI-R2), para o contexto brasileiro. Os passos para a operacionalização dessa etapa da pesquisa foram: tradução, tradução reversa, síntese das versões traduzidas, avaliação por especialistas, avaliação pelo público-alvo e estudo piloto. A amostra para o levantamento de evidências de validade semântica foi de 40 alunos, com idade média de 10 anos e 8 meses $(D P=2,3)$, sendo $75 \%$ do sexo feminino e $25 \%$ do sexo masculino. A amostra para o estudo piloto foi de 84 alunos, com idade média de 10 anos e 7 meses $(D P=2,7)$, sendo que o sexo feminino representou $58,3 \%$ da amostra e o masculino $41,7 \%$. Os alunos de ambas as amostras pertenciam ao ensino fundamental público do estado do Paraná. Foi utilizada a estatística descritiva para o tratamento dos dados e os resultados indicaram uma adequação da escala em relação a análise dos itens, sendo que a avaliação de juízes se mostrou positiva, permitindo alcançar índices de concordância considerados adequados. Por fim, foram discutidas as convergências e discrepâncias entre as versões traduzida/ original e a adaptada para o contexto brasileiro.

Palavras-chave: evidências de validade, estilos de aprendizagem, avaliação psicológica.

\section{ABSTRACT - Translation, Adaptation and Validity Evidence for the Thinking Styles Inventory - Revised II (TSI-R2) in Brazil}

The purpose of this study was to translate, adapt and analyze content validity evidence for the Thinking Styles Inventory-Revised II (TSI-R2) for the Brazilian context. The steps for this stage of the research were: translation, reverse translation, synthesis of translated versions, evaluation by specialists, evaluation by the target audience and pilot study. The collection sample for evidence of semantic validity was 40 students, mean age of 10 years and 8 months ( $S D=2.3$ ), $75 \%$ female and $25 \%$ male. The sample for the pilot study was 84 students, with an average age of 10 years and 7 months $(S D=2.7)$. Females represented $58.3 \%$ of the sample and males $41.7 \%$. Both student samples attended public elementary schools in the state of Paraná. Descriptive statistics were used to treat the data and results indicated the scale's adequacy in relation to the items' analysis, and the judges' evaluation was positive, allowing agreement indexes considered appropriate. Finally, convergences and discrepancies between the translated/original and the adapted versions for the Brazilian context are discussed.

Keywords: validity evidence, learning styles, psychological evaluation.

\section{RESUMEN - Traducción, Adaptación y Evidencias de Validez Thinking Styles Inventory Estilos de Pensamiento - II Revisado (TSI-R2) en Brasil}

El objetivo del presente estudio fue traducir, adaptar y analizar evidencias de validez de contenido en el Thinking Styles Inventory Revised II (TSI-R2), en el contexto brasileño. Las etapas de esta investigación fueron: traducción, traducción inversa, síntesis de versiones traducidas, evaluación por especialistas, evaluación del público objetivo y estudio piloto. La muestra para el levantamiento de evidencias de validez semántica estuvo compuesta por 40 alumnos, con una edad media de 10 años y 8 meses $(D P=2,3)$ siendo $75 \%$ de sexo femenino y $25 \%$ de sexo masculino. La muestra para el estudio piloto fue de 84 alumnos, con promedio de 10 años y 7 meses $(D P=2,7)$, siendo un 58,3\% femenino y un 41,7\% masculino. Los alumnos de ambas muestras eran de Enseñanza Primaria de escuelas públicas del estado de Paraná. Se utilizaron estadísticas descriptivas para el tratamiento de datos y los resultados indicaron una adecuación de la escala con relación al análisis de los ítems, y la evaluación de los jueces se mostró positiva, permitiendo alcanzar índices de concordancia adecuados. Finalmente, se discutieron las convergencias y discrepancias entre las versiones traducida/ original y la adaptada para el contexto brasileño.

Palabras clave: evidencias de validez, estilos de aprendizaje, evaluación psicológica.

${ }^{1}$ Endereço para correspondência: Laboratório de Avaliação e Pesquisa Psicológica/LAPPSIC. Depto de Psicologia e Psicanálise. Centro de Ciências Biológicas. Universidade Estadual de Londrina. Campus Universitário. Rodovia Celso Garcia Cid, PR 445, km 380, Cx. Postal 6001, 86051-990, Londrina-PR. Tel.: (43) 3371-4397. E-mail: katyauel@omail.com

Apoio financeiro: CNPq e CAPES. 
Ao trazer para outra realidade um instrumento psicométrico, é necessário que algumas etapas sejam cumpridas de forma a garantir que ele possa mensurar adequadamente o fenômeno para o qual foi construído (Borsa, Damásio, \& Bandeira, 2012). Sob essa perspectiva, o propósito do presente estudo é o de relatar os passos que foram seguidos para que Inventário de Estilos de Pensamento fosse adaptado para o Brasil, especialmente no que se refere ao estudo de suas evidências de validade de conteúdo.

As pesquisas desenvolvidas sobre a cognição humana têm buscado compreender o modo como as pessoas pensam, interpretam e percebem o mundo. $\mathrm{Na}$ teoria do processamento da informação, avaliam-se as capacidades intelectuais humanas, buscando-se compreender as estratégias e os modelos empregados pelos indivíduos para a resolução de problemas (Sternberg, 2000). Sob essa perspectiva, o estilo intelectual se refere à maneira preferida utilizada pelo indivíduo para tomar decisões, usar suas habilidades, resolver problemas e processar informações (Fan \& Zhang, 2014; Wang \& Tseng, 2014; Zhang \& Sternberg, 2005).

No que concerne ao campo dos estilos, há que se mencionar o fato de até recentemente haver certa discrepância entre as nomenclaturas existentes para a sua conceituação (Oliveira, Santos, \& Scacchetti, 2016; Zhang \& Sternberg, 2005). Tal fato se deve a diversos fatores, dentre eles, a ausência de padronização dos métodos e instrumentos utilizados nos estudos sobre o tema, bem como a falta de uma linguagem comum entre psicólogos e pesquisadores da área, acarretando diversas formas de se referir aos estilos, como, por exemplo, estilos de aprendizagem, cognitivos, de mente, de pensar, modos de pensar, dentre outros. Mais recentemente, o termo "estilos intelectuais" tem sido considerado o mais completo teoricamente, sendo utilizado como um termo "guarda-chuva" que engloba as demais terminologias anteriormente empregadas (Fan \& Zhang, 2014; Zhang, 2011, 2015).

Dentre as diversas classificações existentes na literatura acerca dos estilos intelectuais, o modelo estruturado a partir da Teoria do Autogoverno Mental faz analogia entre os estilos e as formas de governo da sociedade. Usando metaforicamente a palavra "governo", entende-se que, assim como há diversas formas de governar uma sociedade, haveria também diversas formas de governarmos nossas próprias atividades (Zhang \& Sternberg, 2005). A Teoria do Autogoverno Mental remete a existência de 13 estilos, divididos em cinco dimensões. A primeira dimensão (função) abrange os estilos legislativo, executivo e judicial. A segunda dimensão (forma) se refere aos estilos hierárquico, monárquico, oligárquico e anárquico. A terceira dimensão (nível) diz respeito aos estilos global e local. A quarta dimensão (espaço) abrange os estilos interno e externo, enquanto a quinta dimensão (tendência) remete aos estilos liberal e conservador (Emir, 2013; Fan \& Zhang, 2014).
Na primeira dimensão (função), o estilo legislativo diz respeito a pessoas criativas que gostam de escolher suas próprias atividades. Por sua vez, o estilo executivo remete a pessoas que preferem seguir instruções claras e regras na realização de suas atividades, enquanto o estilo judicial abrange pessoas que gostam de problemas em que seja possível avaliar o desempenho dos demais. $\mathrm{Na}$ segunda dimensão (forma), pessoas no estilo hierárquico costumam organizar suas atividades seguindo uma hierarquia de prioridades, enquanto pessoas no estilo monárquico, preferem focar em uma atividade por vez, tendo poucas prioridades. Por sua vez, pessoas no estilo oligárquico costumam realizar várias tarefas ao mesmo tempo sem estabelecer prioridades, enquanto pessoas no estilo anárquico dão preferência para tarefas flexíveis, não estabelecendo objetivos (Zhang, 2015).

$\mathrm{Na}$ terceira dimensão (nível), o estilo global abarca pessoas com ideias abstratas e que não apreciam detalhes, enquanto o estilo local diz respeito à preferência por ideias concretas e tarefas com detalhes. Em relação à quarta dimensão (espaço), pessoas no estilo interno preferem trabalhar sozinhas, enquanto pessoas no estilo externo dão preferência por atividades em equipe. Por fim, na quinta dimensão (tendência), pessoas no estilo liberal gostam de tarefas que não lhe são familiares, apreciando a mudança, enquanto o estilo conservador diz respeito a pessoas que gostam de seguir regras e manter procedimentos fixos na realização de suas atividades (Zhang, Sternberg, \& Fan, 2013; Zhang, 2015).

Além da disposição realizada por meio das dimensões, Zhang e Sternberg (2005) classificaram os treze estilos intelectuais em três tipos, sendo eles, o Tipo I (legislativo, judicial, liberal, global e hierárquico), Tipo II (executivo, conservador, local e monárquico) e o Tipo III (oligárquico, anárquico, interno e externo). Nesse sentido, o Tipo I abrange os estilos que denotam maior criatividade, originalidade e autonomia, exigindo níveis mais altos de complexidade cognitiva. O Tipo II demonstra preferência por tarefas mais estruturadas, que exigem baixo nível de complexidade cognitiva. Por fim, o Tipo III pode apresentar características de ambos os tipos I e II, sendo seu uso dependente das exigências estilísticas da situação e da atividade a ser realizada (Cheng, Hu, \& Sin, 2016; Fan, 2016; Nielsen, 2014; Zhang et al., 2013).

Zhang $(2013,2015)$ afirma que os estilos intelectuais são modificáveis e podem ser ensinados e desenvolvidos ao longo da vida, pois dependem da interação entre o homem e o ambiente. Apesar de não serem classificados como bons ou ruins, alguns estilos podem ser utilizados preferencialmente em uma situação de aprendizagem, podendo, na perspectiva educacional, auxiliar no desempenho escolar do aluno. Para tanto, o próprio aluno deve fazer uso do estilo que melhor condiz com a sua forma de aprender. Nessa direção, cabe elucidar o papel do professor e das instituições de ensino quanto ao 
reconhecimento e uso dos estilos intelectuais como facilitadores no processo de ensino e aprendizagem (Gomes, Marques, \& Golino, 2014). Além disso, ressalta-se que os estudos acerca dos estilos intelectuais têm se desenvolvido não apenas no ambiente acadêmico, como também em outros contextos (Fan, 2016).

Conforme descrito por Zhang e Sternberg (2005), o Inventário de Estilos de Pensamento foi criado com o objetivo de operacionalizar a Teoria do Autogoverno Mental. O instrumento possui origem estadunidense e apresenta estudos que evidenciam sua validade para aquele contexto no ensino superior e médio, com coeficiente do alfa de Cronbach para as dimensões da escala variando de 0,70 a 0,80 .

Elaborado inicialmente por Sternberg e Wagner (1992), o Inventário de Estilos de Pensamento apresentava 104 itens organizados em uma estrutura teórica de 13 estilos: legislativo, executivo, julgamento, global, local, liberal, conservador, hierárquico, monárquico, oligárquico, anárquico, interno e externo. Anos mais tarde foi realizada uma revisão do TSI, por Sternberg, Wagner e Zhang (2003), passando a conter 65 itens divididos entre os 13 estilos da Teoria do Autogoverno Mental. Em uma nova revisão do instrumento proposta por Sternberg et al. (2007), o Inventário de Estilos de Pensamento recebeu a denominação Revisado II (TSI-R2). Essa versão também contém 65 itens e 13 dimensões (Zhang, 2011).

$\mathrm{Na}$ literatura científica, os estudos sobre estilos que utilizam o Thinking Styles Inventory ou Inventário de Estilos de Pensamento (TSI, Sternberg \& Wagner, 1992; ETI-R e TSI-R2, Sternberg et al., 2003, 2007) se concentram, sobretudo na literatura estrangeira (Bernardo et al., 2009; Cheng et al., 2016; Cheng, Zhang, \& Hu, 2016; Emir, 2013; Esmer \& Altun, 2016; Fan, 2016; Fan \& Zhang, 2014; Hassan, 2014; Pusina, 2014). Nacionalmente, destacam-se alguns estudos publicados nos últimos anos (Gomes, et al., 2014; Inácio, Oliveira, \& Santos, no prelo; Oliveira, Inácio, \& Buriolla, 2016). Em ambos os casos, as pesquisas são predominantemente realizadas no contexto do ensino superior, sendo a temática dos estilos pouco explorada nas demais etapas de ensino, inclusive no ensino fundamental.

Especificamente com o ensino fundamental, o estudo de González-Pienda et al. (2004), buscou analisar a estrutura fatorial do Thinking Styles Inventory de Sternberg e Wagner (1992) em 2.698 estudantes do ensino fundamental da Espanha. A partir da pesquisa, os autores corroboraram a Teoria do Autogoverno Mental, na qual os estilos são organizando segundo 13 dimensões. Verificaram ainda que os 13 estilos se combinam de tal modo que possibilitam a existência de três tipos gerais de estilos, sendo eles: legislativo/judicial, executivo/conservador e oligárquico/externo.

Nacionalmente, Oliveira, Santos e Scacchetti (2016) analisaram as diferenças, considerando o ano escolar em relação aos estilos intelectuais. Utilizando o Inventário de Estilos de Pensamento (TSI-R2) de Sternberg et al. (2007), os resultados indicaram que os estudantes do $2^{\circ}$ ao $4^{\circ}$ ano pontuaram mais nos estilos legislativo, executivo, conservador, monárquico, oligárquico, interno e externo. Já os estudantes do $5^{\circ}$ ao $7^{\circ}$ ano se saíram melhor nos estilos judicial, global, local, liberal, anárquico e hieráquico, sendo que, neste último, o resultado foi semelhante entre o grupo de $5^{\circ}$ ao $7^{\circ}$ ano e o grupo de $8^{\circ}$ e $9^{\circ}$ ano. Diante desses resultados fica evidente a diferenciação existente entre os estilos de pensamento, ou seja, o modo como o indivíduo prefere utilizar suas habilidades em cada etapa de ensino, mesmo dentro de um contexto maior que, nesse caso, seria o ensino fundamental. As autoras trazem ainda a importância da validação desse instrumento, bem como de futuros estudos com os quais os resultados ora emergidos possam ser comparados.

Ao considerar a necessidade de ampliação dos estudos acerca dos estilos intelectuais, especialmente em contexto nacional, tendo em vista que os estudos dessa temática se mostram incipientes e ainda pelo fato de que o mapeamento dos estilos em sala de aula pode auxiliar no momento da aprendizagem e contribuir no desempenho escolar dos alunos, o presente estudo buscou traduzir, adaptar e analisar evidências de validade de conteúdo para o Thinking Styles Inventory - Revised II (TSI-R2) (Sternberg, Wagner, \& Zhang 2007), para o contexto brasileiro. Com isso espera-se contribuir com o desenvolvimento da área de avaliação psicológica direcionada à mensuração de processos cognitivos e de aprendizagem, disponibilizando uma medida de estilos intelectuais, já utilizada em âmbito mundial.

\section{Método}

\section{Participantes}

A tradução do TSI-R2 contou com a participação de dois juízes independentes, ambos bilíngues português-inglês, do sexo feminino, com formação acadêmica e idades entre 35 e 45 anos, sendo um deles nativo (de modo a garantir que um nativo pudesse também efetivar essa tradução). Esses juízes efetivaram a tradução. A retrotradução contou com um juíz nativo na língua inglesa (norte americana), que realizou a tradução do instrumento novamente para o inglês. Após essa etapa, participaram outros dois juízes independentes especialistas da língua, ambos profissionais que atuam na área de tradução de instrumentos em língua inglesa estudados na realidade brasileira, também do sexo feminino, com formação acadêmica e idades entre 40 e 50 anos.

Para avaliação do conteúdo dos itens e dimensões representativas deles, participaram cinco juízes especialistas, sendo três doutores e dois mestres da área de processos cognitivos e de aprendizagem, todos do sexo feminino e idades que variaram entre 40 e 60 anos. Esses juízes constituíram o grupo de especialistas acerca da 
compreensão do construto. Os juízes com título de doutor eram professores universitários na área de psicologia e pertencentes a programas de pós-graduação Stricto Sensu de diferentes universidades.

No que tange à busca de evidências de validade semântica para a escala participaram 40 alunos de duas escolas (uma escola pública municipal e outra estadual), sendo cinco alunos $(12,5 \%)$ de cada ano escolar, considerando do $2^{\circ}$ ao $9^{\circ}$ anos que avaliaram a primeira tradução do instrumento. A média de idade foi de 10 anos e 8 meses $(D P=2,3)$, a idade mínima foi de 7 anos e a máxima de 15 anos, sendo $75 \%(n=30)$ do sexo feminino e $25 \%$ $(n=10)$ do masculino.

Para o estudo piloto, participaram 84 alunos provenientes de quatro escolas (duas públicas municipais e duas estaduais), sendo aproximadamente 10 de cada ano escolar, do $2^{\circ}$ ao $9^{\circ}$ anos. No caso do $2^{\circ}$ ano, foram aplicados em $14(16,7)$ alunos, para se ter uma ideia mais clara acerca da compreensão da instrução de aplicação. Nos demais anos, a distribuição foi de $10(11,9 \%)$ alunos por ano. Alunos do $1^{\circ}$ ano não foram incluídos na amostra, por não estarem totalmente alfabetizados. A média de idade foi de 10 anos e 7 meses $(D P=2,7)$, a idade mínima foi de 7 e a máxima de 15 anos, sendo que o sexo feminino representou $58,3 \%(n=49)$ da amostra e o masculino $41,7 \%(n=35)$. Cabe esclarecer que os alunos que participaram do procedimento de validade semântica não participaram do estudo piloto.

\section{Instrumento}

Para a realização do presente estudo foi empregado o Inventário de Estilos de Pensamento - Thinking Styles Inventory - TSI-R2 (Sternberg et al., 2007). Esse instrumento apresenta 65 itens distribuídos em 13 estilos, em que o aluno deverá ler cada uma das frases e responder em uma escala Likert de sete pontos o quanto a situação apresentada se aplica a ele, sendo: "De jeito nenhum" (1 ponto), "Não muito bem" (2 pontos), "Um pouco" (3 pontos), "Bem de alguma forma" (4 pontos), "Bem" (5 pontos), "Muito bem" (6 pontos) e "Extremamente bem" (7 pontos) (Zhang, 2011). A Tabela 1 indica os 13 estilos que constam no instrumento e apresenta como exemplo frases referentes a cada um deles para melhor compreensão.

Tabela 1

Estilos de Pensamento com Exemplos

\begin{tabular}{l|l}
\hline \multicolumn{1}{c|}{ Estilos } & \multicolumn{1}{c}{ Exemplos } \\
\hline Legislativo & Eu gosto de brincar com minhas ideias e ver quão longe elas vão. \\
\hline Executivo & Sou cuidadoso(a) em usar uma forma/jeito adequado para resolver qualquer problema. \\
\hline Judicial & Eu gosto de verificar e avaliar diferentes pontos de vista. \\
\hline Global & $\begin{array}{l}\text { Eu me importo mais com a ideia geral de uma tarefa que tenho que fazer do que sobre os } \\
\text { detalhes da mesma. }\end{array}$ \\
\hline Local & $\begin{array}{l}\text { Ao discutir ou escrever sobre um assunto, eu acho que os detalhes e fatos são mais importantes } \\
\text { que a ideia geral. }\end{array}$ \\
\hline Liberal & Quando eu enfrento um problema, eu prefiro tentar novas estratégias ou métodos para resolvê-lo. \\
\hline Conservador & Quando eu estou no comando de alguma coisa, eu gosto de seguir modelos e ideias usadas no passado. \\
\hline Hierárquico & Ao trabalhar em uma tarefa, eu posso ver como as partes se relacionam com o objetivo geral da tarefa. \\
\hline Monárquico & Se há diversas coisas importantes para fazer, eu foco na coisa mais importante pra mim e ignoro o resto. \\
\hline Oligárquico & Prefiro trabalhar em um projeto ou tarefa que é aceitável e aprovado pelos meus colegas. \\
\hline Anárquico & $\begin{array}{l}\text { Eu tendo a enfrentar vários problemas ao mesmo tempo, porque eles são muitas vezes } \\
\text { igualmente urgentes. }\end{array}$ \\
\hline Interno & Quando tento tomar uma decisão, eu confio em minha própria opinião sobre a situação. \\
\hline Externo & Em uma atividade escolar, eu gosto de combinar as minhas próprias ideias com as dos outros. \\
\hline
\end{tabular}

Para a avaliação dos itens, foi enviado aos juízes especialistas, além da taxionomia de cada dimensão, um formulário no qual o juiz avaliador pudesse assinalar se concordava ou discordava que o item se adequava à dimensão proposta, como indicativo da pertinência do item. Utilizou-se um índice de concordância (IC) de $80 \%$, conforme aponta a literatura científica (ver Primi, Muniz, \& Nunes, 2009; Urbina, 2007).

Além disso, empregou-se com os estudantes uma entrevista na qual o aplicador assinalava o grau de compreensão do aluno sobre cada um dos itens em uma escala Likert de quatro pontos, variando de 4 (entendeu completamente), 3 (entendeu parcialmente), 2 (entendeu outra coisa diferente) e 1 (não entendeu).

\section{Critérios Adotados nos Procedimentos}

No que preconiza a International Test Comission ITC (2010) estão descritas etapas para que se realize a adaptação de instrumentos, quais sejam, tradução do instrumento para o contexto nacional, tradução reversa, 
avaliação da síntese por especialistas da área do construto, avaliação do instrumento pelo público ao qual o instrumento será destinado, estudo piloto e análise da estabilidade da estrutura, isto é, análises fatoriais exploratória e confirmatória. No presente estudo, serão apresentados os dados inerentes a essa descrição até a etapa do estudo piloto.

\section{Procedimentos de Tradução}

Inicialmente, o TSI-R2 foi trazido do inglês para o português por dois tradutores bilíngues, sendo que um deles era nativo no idioma inglês. O TSI-R2 foi encaminhado via e-mail para os dois tradutores, para os quais foi solicitado que o instrumento fosse traduzido do inglês para o português.

\section{Procedimento de Retrotradução - Backtranslation}

Nessa etapa, a versão do TSI-R2 foi retrotraduzida para o idioma inglês, o procedimento foi realizado por um tradutor bilíngue, sendo este nativo no idioma inglês (EUA). É importante salientar que foram seguidas as recomendações de Borsa, Damásio e Bandeira (2012).

\section{Procedimento de Avaliação por Juízes Especialistas}

O TSI-R2 também foi avaliado por juízes especialistas. Estes avaliaram a adequação da formulação dos itens. Do mesmo modo, o instrumento foi enviado por e-mail para os juízes e foi solicitado a eles que avaliassem a adequação da formulação dos itens que seriam aplicados a escolares brasileiros do $2^{\circ}$ ao $9^{\circ}$ ano do ensino fundamental.

\section{Procedimento de Busca de Validade Juízes e Público-Alvo}

Nessa etapa, o instrumento, após ter passado pela avaliação dos juízes especialistas e feita a retrotradução, duas etapas foram realizadas. Na primeira, o instrumento foi enviado por e-mail aos juízes especialistas acerca da compreensão do construto e, aos avaliadores, foi enviada uma taxionomia de cada dimensão (13 no total) que compunha o instrumento. Foi enviado um formulário no qual o juíz avaliador pudesse assinalar se concordava (C) ou não concordava (NC) que o item se adequava à dimensão proposta. Cabe esclarecer que não foi dada a opção de o juiz alocar item de uma dimensão para a outra quando não concordava.

Após esse procedimento, o instrumento foi aplicado nos alunos de forma coletiva em pequenos grupos separados por ano escolar, esses alunos foram selecionados aleatoriamente dentre aqueles cujos pais e responsáveis consentiram na participação. Após a aplicação coletiva nos grupos, cada criança foi chamada individualmente para passar pelo seguinte procedimento: foi feita a leitura de cada item/pergunta do instrumento. Para cada item, o pesquisador questionou: "Você entendeu o que foi perguntado?" Aguardou-se a resposta da criança (sim ou não); se fosse positiva. o pesquisador dizia: "Então me explique o que você entendeu". A partir da resposta da criança, o pesquisador julgou se o item foi ou não compreendido. Em um formulário, o aplicador assinalava o grau de compreensão do aluno sobre o item em escala Likert de quatro pontos, variando se entendeu completamente (4), entendeu parcialmente (3), entendeu outra coisa ou sentido diferente (2) e não entendeu (1).

\section{Procedimento do Estudo Piloto}

Nessa etapa, foi realizada a coleta nas escolas públicas, tendo já a aprovação do comitê de ética em pesquisa (conforme resolução 196/96 e seus complementares). Os pais dos alunos participantes (aproximadamente 84, dos $2^{\circ}$ ao $9^{\circ}$ anos) assinaram um Termo de Consentimento Livre e Esclarecido, bem como os alunos, autorizando a realização da coleta. O procedimento de coleta dos dados foi realizado em dia e horário previamente agendados nas instituições, tendo duração aproximada de 30 minutos. $\mathrm{O}$ aplicador convidava os alunos ora indicados por seus professores a se dirigirem em sala cedida pela instituição escolar. Ao chegar, os alunos sentavam e lhes era dito que estavam ali para responder a um questionário com questões sobre o modo como eles estudam e aprendem. Foi solicitado também que eles fossem sinceros ao responder e que não havia respostas certas ou erradas.

\section{Análise de Dados}

Os dados foram organizados em planilhas e submetidos à estatística descritiva, visando atender ao objetivo do presente estudo. Foram levantados dados de média, desvio padrão, bem como dados percentuais. Para efetivar a análise dos dados do julgamento dos juízes, empregou-se a estimativa do índice de concordância (IC) obtida entre os avaliadores independentes. Assim, tomou-se, como critério para que o item fosse considerado satisfatório, o valor de concordância de $80 \%$, como critério para se estabelecer a pertinência do item (ver Pasquali, 2010; Primi, Muniz, \& Nunes, 2007).

\section{Resultados}

Os resultados serão apresentandos na sequência descrita na parte de procedimento do presente estudo. No que se refere ao procedimento de tradução, observou-se a incoerência entre os tradutores quanto à tradução dos itens. Em relação ao título do instrumento, um tradutor entendeu que seria Inventário de Estilos de Pensamento (Thinking Styles Inventory), o outro, por sua vez, apontou que seria adequado empregar Inventário de Estilos Intelectuais. Por essa razão, um terceiro tradutor foi chamado para somente poder opinar acerca do título e ele entendeu que o mais apropriado seria Inventário de Estilos Intectuais.

Quanto à realização de avaliação por juízes especialistas linguístas o TSI-R2, foi analisado e alguns 
itens sofreram adaptações em sua formulação para que pudessem ficar próprios para os escolares do ensino fundamental. Desse modo, por exemplo, o item 1 na versão apenas traduzida seria: "Eu prefiro lidar com problemas que exijam que me atenha a muitos detalhes", enquanto na versão adaptada ficaria: "Eu prefiro lidar com problemas que exijam que eu preste atenção a muitos detalhes". Outro exemplo, no item 13, versão apenas traduzida seria: "Atenho-me a regras padrão ou maneiras de fazer as coisas", enquanto na versão adaptada ficaria: "Prefiroseguir regras oumaneiras prontas de fazer as coisas".

Para a condução do processo de validade de conteúdo, dois métodos foram selecionados. O primeiro deles, seria a estimativa do índice de concordância (IC) entre os juízes independentes, dessa forma, adotou-se o seguinte critério: para que o item fosse considerado satisfatório, o valor de concordância entre os juízes deveria ser pelo menos de $80 \%$. A Tabela 2 mostra a porcentagem de concordância entre os juízes em cada dimensão avaliada.

Tabela 2

Índice de Concordância entre os Juízes em cada Dimensão do TSI-R2

\begin{tabular}{|c|c|c|c|c|c|c|c|}
\hline \multirow{2}{*}{ Estilo } & \multirow{2}{*}{ Item } & \multicolumn{5}{|c|}{ Juiz } & \multirow{2}{*}{ \% de Concordância } \\
\hline & & J1 & $\mathrm{J} 2$ & J3 & $\mathrm{J} 4$ & J5 & \\
\hline Legislativo & $\begin{array}{c}5 \\
10 \\
14 \\
32 \\
49 \\
\end{array}$ & $\begin{array}{l}C \\
C \\
C \\
C\end{array}$ & $\begin{array}{c}\mathrm{C} \\
\mathrm{NC} \\
\mathrm{C} \\
\mathrm{C}\end{array}$ & $\begin{array}{l}C \\
C \\
C \\
C\end{array}$ & $\begin{array}{l}C \\
C \\
C \\
C\end{array}$ & $\begin{array}{l}C \\
C \\
C \\
C\end{array}$ & $\begin{array}{c}100 \\
80 \\
100 \\
100 \\
100 \\
\end{array}$ \\
\hline \multirow{5}{*}{ Executivo } & 8 & $\mathrm{C}$ & $\mathrm{C}$ & $\mathrm{C}$ & $\mathrm{C}$ & $\mathrm{C}$ & 100 \\
\hline & 11 & $\mathrm{C}$ & $\mathrm{C}$ & C & $\mathrm{C}$ & $\mathrm{C}$ & 100 \\
\hline & 12 & $\mathrm{C}$ & $\mathrm{C}$ & $\mathrm{C}$ & $\mathrm{C}$ & $\mathrm{C}$ & 100 \\
\hline & 31 & $\mathrm{C}$ & $\mathrm{C}$ & $\mathrm{C}$ & $\mathrm{C}$ & C & 100 \\
\hline & 39 & $\mathrm{C}$ & $\mathrm{C}$ & $\mathrm{C}$ & $\mathrm{NC}$ & $\mathrm{C}$ & 80 \\
\hline \multirow{5}{*}{ Judicial } & 20 & $\mathrm{C}$ & $\mathrm{C}$ & $\mathrm{C}$ & $\mathrm{C}$ & $\mathrm{C}$ & 100 \\
\hline & 23 & $\mathrm{C}$ & $\mathrm{C}$ & $\mathrm{C}$ & $\mathrm{C}$ & $\mathrm{C}$ & 100 \\
\hline & 42 & $\mathrm{C}$ & $\mathrm{C}$ & $\mathrm{C}$ & $\mathrm{C}$ & $\mathrm{C}$ & 100 \\
\hline & 51 & $\mathrm{C}$ & $\mathrm{C}$ & $\mathrm{C}$ & $\mathrm{C}$ & $\mathrm{C}$ & 100 \\
\hline & 57 & $\mathrm{C}$ & $\mathrm{C}$ & $\mathrm{C}$ & $\mathrm{C}$ & $\mathrm{C}$ & 100 \\
\hline \multirow{5}{*}{ Global } & 7 & $\mathrm{C}$ & $\mathrm{C}$ & $\mathrm{C}$ & $\mathrm{C}$ & $\mathrm{C}$ & 100 \\
\hline & 18 & $\mathrm{C}$ & NC & $\mathrm{C}$ & C & C & 80 \\
\hline & 38 & $\mathrm{C}$ & C & $\mathrm{C}$ & $\mathrm{C}$ & $\mathrm{C}$ & 100 \\
\hline & 48 & $\mathrm{C}$ & $\mathrm{C}$ & $\mathrm{NC}$ & $\mathrm{C}$ & $\mathrm{C}$ & 80 \\
\hline & 61 & $\mathrm{C}$ & $\mathrm{C}$ & C & C & $\mathrm{C}$ & 100 \\
\hline \multirow{5}{*}{ Local } & 1 & $\mathrm{C}$ & $\mathrm{C}$ & $\mathrm{C}$ & $\mathrm{C}$ & C & 100 \\
\hline & 6 & $\mathrm{C}$ & $\mathrm{C}$ & $\mathrm{C}$ & C & $\mathrm{C}$ & 100 \\
\hline & 24 & $\mathrm{C}$ & $\mathrm{C}$ & $\mathrm{C}$ & $\mathrm{C}$ & $\mathrm{C}$ & 100 \\
\hline & 44 & $\mathrm{C}$ & $\mathrm{C}$ & $\mathrm{C}$ & $\mathrm{C}$ & $\mathrm{C}$ & 100 \\
\hline & 62 & $\mathrm{C}$ & $\mathrm{C}$ & $\mathrm{C}$ & $\mathrm{C}$ & $\mathrm{C}$ & 100 \\
\hline \multirow{5}{*}{ Liberal } & 45 & $\mathrm{C}$ & $\mathrm{C}$ & $\mathrm{C}$ & $\mathrm{C}$ & $\mathrm{C}$ & 100 \\
\hline & 53 & $\mathrm{C}$ & $\mathrm{C}$ & $\mathrm{C}$ & $\mathrm{C}$ & $\mathrm{C}$ & 100 \\
\hline & 58 & $\mathrm{C}$ & C & $\mathrm{C}$ & C & C & 100 \\
\hline & 64 & $\mathrm{C}$ & $\mathrm{C}$ & $\mathrm{C}$ & C & $\mathrm{C}$ & 100 \\
\hline & 65 & $\mathrm{C}$ & $\mathrm{C}$ & $\mathrm{C}$ & $\mathrm{C}$ & $\mathrm{C}$ & 100 \\
\hline \multirow{5}{*}{ Conservador } & 22 & C & C & C & C & C & 100 \\
\hline & 26 & $\mathrm{C}$ & $\mathrm{C}$ & $\mathrm{C}$ & C & C & 100 \\
\hline & $\begin{array}{l}20 \\
28\end{array}$ & $\mathrm{C}$ & $\mathrm{C}$ & $\mathrm{C}$ & C & C & 100 \\
\hline & 36 & $\mathrm{C}$ & C & C & C & C & 100 \\
\hline & & & & & & & 100 \\
\hline \multirow{5}{*}{ Hierárquico } & 4 & $\mathrm{C}$ & $\mathrm{C}$ & $\mathrm{C}$ & C & C & 100 \\
\hline & 1 & $\mathrm{C}$ & $\mathrm{C}$ & $\mathrm{C}$ & $\mathrm{C}$ & $\mathrm{C}$ & 100 \\
\hline & 33 & $\mathrm{C}$ & $\mathrm{C}$ & $\mathrm{C}$ & $\mathrm{C}$ & $\mathrm{C}$ & 100 \\
\hline & 25 & $\mathrm{C}$ & $\mathrm{C}$ & $\mathrm{C}$ & C & C & 100 \\
\hline & 56 & $\mathrm{C}$ & $\mathrm{C}$ & $\mathrm{C}$ & $\mathrm{C}$ & $\mathrm{C}$ & 100 \\
\hline \multirow{5}{*}{ Monárquico } & 2 & $\mathrm{C}$ & NC & $\mathrm{C}$ & C & $\mathrm{C}$ & 80 \\
\hline & 43 & $\mathrm{C}$ & C & NC & C & C & 80 \\
\hline & 50 & $\mathrm{C}$ & $\mathrm{C}$ & C & C & $\mathrm{C}$ & 100 \\
\hline & 54 & $\mathrm{C}$ & C & $\mathrm{NC}$ & C & C & 80 \\
\hline & 60 & $\mathrm{C}$ & $\mathrm{C}$ & C & C & $\mathrm{C}$ & 100 \\
\hline
\end{tabular}


Tabela 2 (continuação)

Índice de Concordância entre os Juízes em cada Dimensão do TSI-R2

\begin{tabular}{|c|c|c|c|c|c|c|c|}
\hline \multirow{2}{*}{ Estilo } & \multirow{2}{*}{ Item } & \multicolumn{5}{|c|}{ Juiz } & \multirow{2}{*}{ \% de Concordância } \\
\hline & & $\mathrm{J} 1$ & $\mathrm{~J} 2$ & J3 & $\mathrm{J} 4$ & J5 & \\
\hline \multirow{5}{*}{ Oligárquico } & 27 & $\mathrm{C}$ & C & $\mathrm{C}$ & $\mathrm{C}$ & C & 100 \\
\hline & 29 & $\mathrm{C}$ & $\mathrm{NC}$ & C & $\mathrm{C}$ & C & 80 \\
\hline & 30 & $\mathrm{C}$ & C & $\mathrm{NC}$ & $\mathrm{C}$ & C & 80 \\
\hline & 52 & C & C & C & C & C & 100 \\
\hline & 59 & $\mathrm{C}$ & C & $\mathrm{NC}$ & $\mathrm{C}$ & $\mathrm{C}$ & 80 \\
\hline \multirow{5}{*}{ Anárquico } & 16 & $\mathrm{C}$ & $\mathrm{C}$ & $\mathrm{C}$ & $\mathrm{C}$ & $\mathrm{C}$ & 100 \\
\hline & 21 & $\mathrm{C}$ & C & C & $\mathrm{C}$ & C & 100 \\
\hline & 35 & $\mathrm{C}$ & C & C & $\mathrm{C}$ & C & 100 \\
\hline & 40 & $\mathrm{C}$ & $\mathrm{C}$ & C & C & C & 100 \\
\hline & 47 & $\mathrm{C}$ & C & $\mathrm{C}$ & $\mathrm{C}$ & $\mathrm{C}$ & 100 \\
\hline \multirow{5}{*}{ Interno } & 9 & $\mathrm{C}$ & $\mathrm{C}$ & $\mathrm{C}$ & $\mathrm{C}$ & $\mathrm{C}$ & 100 \\
\hline & 15 & $\mathrm{C}$ & C & C & C & C & 100 \\
\hline & 37 & $C$ & C & C & C & C & 100 \\
\hline & 55 & $C$ & C & C & C & C & 100 \\
\hline & 63 & $\mathrm{C}$ & C & C & C & C & 100 \\
\hline \multirow{5}{*}{ Externo } & 3 & $C$ & C & C & $\mathrm{C}$ & C & 100 \\
\hline & 17 & $\mathrm{C}$ & C & C & C & C & 100 \\
\hline & 34 & $\mathrm{C}$ & C & C & C & C & 100 \\
\hline & 41 & $C$ & C & C & C & C & 100 \\
\hline & 46 & C & $\mathrm{C}$ & C & C & C & 100 \\
\hline
\end{tabular}

Nota. $\mathrm{C}=$ concorda; $\mathrm{NC}=$ não concorda

Os juízes não demonstraram discrepâncias na análise dos itens em suas respectivas dimensões. Conforme pode ser observado na Tabela 2, o IC ficou dentro do limite indicado na literatura científica, conforme indicam Primi, Muniz e Nunes (2007).

O segundo método para a busca de evidências de validade de conteúdo foi efetivado visando obter informações sobre a inteligibilidade dos itens da escala pelos alunos. Para tanto, foi empregada pelo avaliador uma escala do tipo Likert de 4 pontos, sendo que 4 expressava o entendimento completo do item (conforme já exposto anteriormente). Considerando que o TSI-R2 tem 65 itens, a pontuação na entrevista poderia chegar a 260. A média de pontos sobre a intelegibilidade obtida com a entrevista foi de 245,6 $(D P=31,2)$. A análise das respostas das crianças pode ser vista na Tabela 3 .

Ao se considerar a pontuação máxima na entrevista (260 pontos), pode-se dizer que a porcentagem de entendimento dos itens ficou em 94,2\%. Uma análise qualitativa acerca das respostas dos alunos do $2^{\circ}$ ano demonstrou que eles apresentaram maior incidência de respostas 1(não entendeu) e 2 (entendeu outra coisa diferente). Hipotetiza-se que talvez porque esses alunos ainda não estejam em um processo de alfabetização já profundamente concretizada.

Com o estudo piloto foi possível observar que os alunos compreenderam as instruções dadas no momento da aplicação e que a aplicação em grupos de 10 alunos pareceu ser adequada para um melhor controle da coleta, como poder dar maior apoio aos alunos, pois, numa coleta menor, é possível observar melhor o comportamento de cada um. Na Tabela 4, constam as médias, desvio padrão e pontuaçãoes máxima e mínima em cada dimensão. Cabe recordar que cada dimensão apresentava cinco itens e que a pontuação no instrumento poderia variar de 1 a 7, dessa forma a pontuação máxima poderia chegar a 35 em cada dimensão.

Tabela 3

Frequências e Porcentagens de Pontos dos Participantes na Entrevista

\begin{tabular}{ccc}
\hline Pontos na entrevista & $n$ de alunos & $\%$ \\
\hline 126,00 & 1 & 2,5 \\
159,00 & 1 & 2,5 \\
175,00 & 1 & 2,5 \\
184,00 & 1 & 2,5 \\
192,00 & 1 & 2,5 \\
251,00 & 1 & 2,5 \\
253,00 & 3 & 7,5 \\
254,00 & 1 & 2,5 \\
255,00 & 3 & 7,5 \\
256,00 & 7 & 17,5 \\
257,00 & 5 & 12,5 \\
258,00 & 6 & 15,0 \\
259,00 & 4 & 10,0 \\
260,00 & 5 & 12,5 \\
\hline Total & 40 & 100,0 \\
\hline
\end{tabular}


Tabela 4

Médias, Desvio Padrão e Pontuaçãoes Mínima e Máxima em cada Dimensão do TSI-R2

\begin{tabular}{cccc}
\hline Estilo & $M$ & $D P$ & $\begin{array}{c}\text { Pontuações Mínima } \\
\text { e Máxima }\end{array}$ \\
\hline Legislativo & 25,6 & 6,4 & $11 / 35$ \\
Executivo & 25,13 & 5,5 & $8 / 35$ \\
Judicial & 22,9 & 6,0 & $9 / 33$ \\
Global & 18,33 & 6,7 & $5 / 33$ \\
Local & 21,1 & 6,2 & $5 / 35$ \\
Liberal & 22,9 & 64 & $8 / 35$ \\
Conservador & 24,3 & 6,6 & $10 / 35$ \\
Hierárquico & 21,2 & 7,3 & $5 / 35$ \\
Monárquico & 25,5 & 5,2 & $11 / 34$ \\
Oligárquico & 22,2 & 7,4 & $5 / 35$ \\
Anárquico & 21,3 & 5,1 & $8 / 34$ \\
Interno & 23,8 & 6,6 & $9 / 35$ \\
Externo & 24,2 & 6,8 & $5 / 35$ \\
\hline
\end{tabular}

Ao se considerar que a metade de 35 (total por dimensão a ser atingido) é 17,5 pode-se considerar que, em todos os casos, os alunos obtiveram uma média correspondente a mais da metade dos pontos possíveis. Há de se mencionar que os alunos do $2^{\circ}$ ano tiveram dificuldade na compreensão da tarefa e, muitas vezes, também demonstraram não compreender o sentido exato da questão, pois apresentaram muitas dúvidas. Novamente, a hipótese de que estar alfabetizado (plenamente por assim dizer) é um fator relevante ao responder a escala. A análise qualitativa do resultado do estudo piloto evidenciou a dificuldade de escolha da resposta na escala Likert, proposta originalmente, com sete gradações de escolha.

\section{Discussão}

A opção pelo estudo do TSI-R2 se deve ao fato de sua vantagem em mensurar tanto os estilos intelectuais quanto os estilos de aprendizagem, visto que segundo a Theory of Mental Self-Government os estilos de aprendizagem estariam contemplados dentro da dimensão maior dos estilos intelectuais, isso porque o termo "estilos intelectuais" tem sido considerado o mais completo teoricamente, sendo utilizado como um termo "guarda-chuva" que engloba as demais terminologias anteriormente empregadas, dentre elas, os estilos de aprendizagem.

Borsa et al. (2012) destacam que construir um instrumento novo seria um processo mais oneroso do que adaptar um já existente. Para os autores, outra vantagem é que a adaptação permite a comparação dos dados obtidos com outras amostras de diferentes contextos, fornecendo uma maior amplitude.

O primeiro procedimento adotado foi o de tradução. Ambos os tradutores demonstraram concordância em suas traduções. A retrotradução assegurou que o procedimento de tradução foi apropriado, não havendo discrepâncias entre as versões. Em relação à análise teórica e semântica de adequação dos itens, houve cuidado para se focalizar a definição constituitiva e também operacional do tipo de tarefa/ação que cada item representava (Medeiros et al., 2015). Para tanto, o pedido aos juízes para que considerassem que os respondentes seriam alunos do ensino fundamental fez a diferença para que várias expressões pudessem ser adaptadas para esse contexto.

No que tange à estimativa do índice de concordância (IC) entre os juízes, o valor da concordância ficou em torno de $80 \%$ (Primi et al., 2009), sendo que a maior parte deles respondeu que concordava que o item correspondia a dimensão indicada. Assim, o envio da taxionomia de cada dimensão auxiliou numa análise mais balizadora, pois todos os juízes seguiram o mesmo parâmetro de definição. Os dados indicam uma adequação da escala em relação à análise dos itens. A avaliação de juízes se mostrou positiva, pois a maioria dos itens foi julgada de modo adequado pelos avaliadores, o que permitiu alcançar índices de concordância considerados adequados.

No que tange à inteligibilidade dos itens da escala pelo público-alvo (ITC, 2010), pode-se dizer que, de modo geral, os alunos demonstraram entender o que estava sendo perguntado no item. $\mathrm{O}$ fato de os alunos do $2^{\circ}$ ano terem tido mais dificuldade para responder, com maior incidência de respostas 1 (não entendeu) e 2 (entendeu outra coisa diferente) parece indicar que o instrumento não estaria apropriado para esse ano escolar. Isso possivelmente seja decorrente de que eles ainda não têm um nível de leitura que permita a compreensão do conteúdo dos itens e, por isso, não conseguiram responder de maneira adequada ao conteúdo solicitado.

Embora os dados do estudo piloto tenham indicado que os alunos, de um modo geral, compreenderam as instruções dadas no momento da aplicação, mais uma vez, verificou-se que os alunos do $2^{\circ}$ ano apresentam dificuldades no entendimento dos itens e também na gradação da possibilidade de escolha da resposta. Diante desse fato, os resultados endossam a ideia de que o instrumento não é apropriado aos alunos do $2 \mathrm{o}$ ano, o que seria uma limitação do presente estudo.

Como limitação, cabe mencionar também que se empregou o índice de concordância dos juízes, dos quais foi apenas pedido que se manifestassem em relação à pertinência ou não do item na dimensão julgada. Dessa forma, não lhes era possível alocar itens em outras dimensões, que julgassem mais adequadas.

Os dados aqui obtidos indicaram que estudos iniciais de averiguação de evidências de validade de uma medida, estendem-se para além dos procedimentos de análise do índice de concordância entre os juízes especialistas da área e do estabelecimento de validação semântica com o público-alvo. Numa perspectiva mais ampla, pode-se considerar que desde o procedimento de tradução, o pesquisador já estaria trabalhando em busca dessa evidência de conteúdo, visto que cada passo indicado no 
ITC (2010) requer cuidado metodológico do pesquisador. Por essa razão, tão importante quanto os procedimentos estatísticos, são as análises de âmbito qualitativo do contexto e da condição comportamental demonstrada pelo público-alvo.

Cabe ressaltar a pertinência do estudo dos estilos em âmbito nacional e internacional, haja vista que o modo como o indivíduo prefere utilizar suas habilidades pode influenciar no desempenho dos estudantes, além de contribuir juntamente com fatores, como a personalidade e a inteligência, para auxiliar nos processos de aquisição do conhecimento. Posto isso, em investigações futuras, sugere-se o estudo da estrutura interna do instrumento e a busca de possíveis relações com as variáveis psicoeducacionais, como a motivação, estratégias de aprendizagem, flexibilidade cognitiva, dentre outras.

\section{Referências}

Bernardo, A. B., Núnez, J. C., González-Pienda, A., Rosário, P., Alvarez, L., González-Castro, P., Valle, A., Rodrígues, S., Cerezo, R., \& Álvarez, D. (2009). Estilos intelectuales y resdimiento académico: uma perspectiva evolutiva. Psicothema, 21(4), 555-561. Recuperado de http://www.psicothema.com/pdf/3670.pdf

Borsa, J. C., Damásio, B. F., \& Bandeira, D. R. (2012). Adaptação e validação de instrumentos psicológicos entre culturas: algumas considerações. Paidéia, 22(53), 423-432. doi: 10.1590/1982-43272253201314

Cheng, S., Hu, X., \& Sin, K. F. (2016). Thinking styles of university deaf or hard of hearing students and hearing students. Research in Developmental Disabilities, 55, 377-387. doi: 10.1016/j.ridd.2016.04.004

Cheng, S., Zhang, L., \& Hu, X. (2016). Thinking styles and university self-effcacy among deaf, hard-of-hearing, and hearing students. Journal of Deaf Studies and Deaf Education, 21(1), 44-53. doi: 10.1093/deafed/env032

Emir, S. (2013). Contributions of teachers' thinking style to critical thinking dispositions (Istanbul-Fatih sample). Educational Science: Theory E Practice, 13(1), 337-347. Recuperado de http://files.eric.ed.gov/fulltext/EJ1016649.pdf

Esmer, E., \& Altun, S. (2016). Teacher candidates' thinking styles: An investigation of various variables. Journal of Education and Training Studies, 4(5), 160-172. doi: 10.11114/jets.v4i5.1392

Fan, J. (2016). The role of thinking styles in career decision-making self-efficacy among university students. Thinking Skills and Creativity, 20, 63-73. doi: 10.1016/j.tsc.2016.03.001

Fan, J., \& Zhang, L. F. (2014). The role of perceived parenting styles in thinking styles. Learning and Individual Differences, 32, 204-211. doi: 10.1016/j.lindif.2014.03.004

Gomes, C. M. A., Marques, E. L. L., \& Golino, H. F. (2014). Validade incremental dos estilos legislativo, executivo e judiciário em relação ao rendimento escolar. Revista Eletrônica de Psicologia, Educação e Saúde, 3(2), 31-46. Recuperado de http://revistaepsi.com/wp-content/ uploads/artigos/2013/Ano3-Volume2-Artigo3.pdf

Hassan, F. S. (2014). Relationship between coping strategies and thinking styles among university students. ASEAN Journal of Psychiatry, 15(1), 14-2. Recuperado de https://www.aseanjournalofpsychiatry.org/index.php/aseanjournalofpsychiatry/article/view/173/138

Inácio, F. F., Oliveira, K. L., \& Santos, A. A. A. (no prelo). Estilos Intelectuais e memória: Análise de alunos com dislexia e transtorno de déficit de atenção/hiperatividade. Estudos de Psicologia de Campinas.

International Test Commission [ITC]. (2010). International Test Commission guidelines for translating and adapting tests. Recuperado de http:// www.intestcom.org/upload/site les/40.pdf

Medeiros, R. K. S., Ferreira Júnior, M. A., Pinto, D. P. S. R., Vitor, A. F., Santos, V. E. P., \& Barichello, E. (2015). Modelo de validação de conteúdo de Pasquali nas pesquisas em enfermagem. Revista de Enfermagem Referência, 4(4), 127-135. doi: 10.12707/RIV14009

Nielsen, T. (2014). Intellectual styles theories: Different types of categorizations and their relevance for practitioners. SpringerPlus, 3(737), 1-10. doi: 10.1186/2193-1801-3-737

Oliveira, K. L., Inácio, A. L. M., \& Buriolla, H. L. (2016). Diferenças considerando ano escolar no ensino fundamental: um estudo com estilos intelectuais. Argumentos Pró-Educação, 1(3), 408-422. Recuperado de http://ojs.univas.edu.br/index.php?journal=argumentospr oeducacao\&page $=$ article\&op $=$ view\&path $\% 5 B \% 5 \mathrm{D}=143$. doi:10.24280/ape.v1i3.143

Oliveira, K. L., Santos, A. A. A., \& Scacchetti, F. A. P. (2016). Medida de estilos de aprendizagem para o ensino fundamental. Psicologia Escolar e Educacional, 20(1), 127-136. doi: 10.1590/2175-3539/2015/0201943

Pasquali, L. (2010). Instrumentação psicológica: Fundamentos e práticas. Porto Alegre, Brasil: Artmed.

Primi, R., Muniz, J., \& Nunes, C. H. S. S. (2009). Definições contemporâneas de validade de testes psicológicos. Em C. S. Hutz (Ed.), Avanços e polêmicas em avaliação psicológica (pp. 243-265). São Paulo: Casa do Psicólogo.

Pusina, A. (2014). Intellectual styles of Bosnian students: A cross-cultural perspective. Procedia - Social and Behavioral Sciences, 141, $298-307$. doi: $10.1016 /$ j.sbspro.2014.05.052

Sternberg, R. J. (2000). Psicologia cognitiva. Porto Alegre: Artes Médicas.

Sternberg, R. J., \& Wagner, R. K. (1992). Thinking Styles Inventory. Unpublished manual, Yale University.

Sternberg, R. J., Wagner, R. K., \& Zhang, L. F. (2003). Thinking styles inventory: Revised. Unpublished: Yale University.

Sternberg, R. J., Wagner, R. K., \& Zhang, L. F. (2007). Thinking styles inventory - Revised II (TSI-R2), Tufts University.

Urbina, S. (2007). Fundamentos da testagem psicológica. Porto Alegre: Artes Médicas.

Wang, T., \& Tsent, Y. (2014). Do thinking styles matter for science achievement and attitudes toward science class in male and female elementary school students in taiwan an? International Journal of Science and Mathematics Education, 13(3), 515-533. doi: 10.1007/s10763013-9503-Z

Zhang, L. F. (2015). Fostering successful intellectual styles for creativity. Asia Pacific Educational Review, 16(2), 183-192. doi: 10.1007/s12564015-9378-5 
Zhang, L. F. (2011). The developing field of intellectual styles: Four recent endeavours. Learning and Individual Differences, 21(3), $311-318$. doi: 10.1016/j.lindif.2010.11.018

Zhang, L. F. (2013). The malleability of intellectual styles. New York: University Press.

Zhang, L., \& Sternberg, R. J. (2005). A threefold model of intellectual styles. Educational Psychology Review, 17(1), 1-53. doi: 10.1007/s10648005-1635-4

Zhang, L., Sternberg, R. J., \& Fan, J. (2013). Revisiting the concept of "style match". British Journal of Educational Psychology, 83(2), $225-237$. doi: org/10.1111/bjep.12011

\section{Sobre as autoras}

Katya Luciane de Oliveira é psicóloga, mestre em Psicologia pelo Programa de Estudos de Pós-graduação stricto sensu em Psicologia da Universidade São Francisco, doutora em Psicologia, Desenvolvimento Humano e Educação pela Faculdade de Educação da Unicamp e professora do Programa de Mestrado e Doutorado em Educação e do Programa de Pós-Graduação em Psicologia ambos da Universidade Estadual de Londrina. Bolsista Produtividade Nível 2/CNPq.

Acácia Aparecida Angeli dos Santos é psicóloga, doutora em Psicologia Escolar e do Desenvolvimento Humano pela USP, docente da graduação no curso de psicologia e no Programa de Pós-graduação stricto sensu em Psicologia, da Universidade São Francisco-SP e bolsista produtividade do CNPq.

Amanda Lays Monteiro Inácio é psicóloga, mestre em Educação pelo Programa de Pós-graduação stricto sensu em Educação da Universidade Estadual de Londrina e doutoranda em Psicologia pelo Programa de Pós-graduação stricto sensu em Psicologia da Universidade São Francisco. 